\title{
Color Recognition System for Visually Impaired Persons
}

\author{
L. Dhamini ${ }^{1}$ and D. Bhanuprakash ${ }^{2}$ \\ ${ }^{1}$ M. Tech student, CVR College of Engineering/ECE Department, Hyderabad, India \\ Email: dhamini94@gmail.com \\ ${ }^{2}$ Assoc. Professor, CVR College of Engineering/ECE Department, Hyderabad, India \\ Email: pbhanududi@gmail.com
}

\begin{abstract}
This paper focuses on assisting tools for visually impaired persons. There is an ongoing research on the HumanComputer-Interaction (HCI) which focuses mainly on the use of computer technology, especially on the interaction between the people and the computer. Visually impaired persons are facing many problems in their day-to-day life as everything around us is encoded visually. This paper aims to propose a camera-based system to identify different colors of clothes. This system integrates a camera, a computer and audio output device for the description of clothing colors. The clothing colors are described verbally to visually impaired person.
\end{abstract}

Index Terms: Camera, Audio Output Device, Visually Impaired.

\section{INTRODUCTION}

This paper aims at designing and developing a device for visually impaired person's individuals as from past few years we can see a rapid growth of them among our population. Even though there is an increase in the many aspects of this technology, but the society is not much interested in designing and developing the applications for such group of people [1]. Many better tools can be developed which are helpful for visually impaired persons and make their life easy to go. Traditionally, such tools which are in the market are heavy and very expensive. For which visually impaired individuals have to be dependent on others for handling the device. In order to reduce this problem, we need to develop on-board capability device and which can also be used for several applications.

Apart from many other assistive tools, this paper aims to develop a color recognition system which gives benefits to the visually impaired person through which they can recognize the color of the clothes [2] and they can decide whether this color of clothes can be worn together or not, according to the norms of color matching [2]. In this system, camera is mounted on the system, for capture the image using some image processing techniques [3].

An Audio output module will be used to give the information about the color of cloth. This is independent and cost-effective assistive system. In this system single board computer systems like Raspberry pi is used for processing the color of the cloth. This scheme is very useful for the people who are visually impaired as well as elderly to make their life more accessible.

\section{A. Block Diagram}

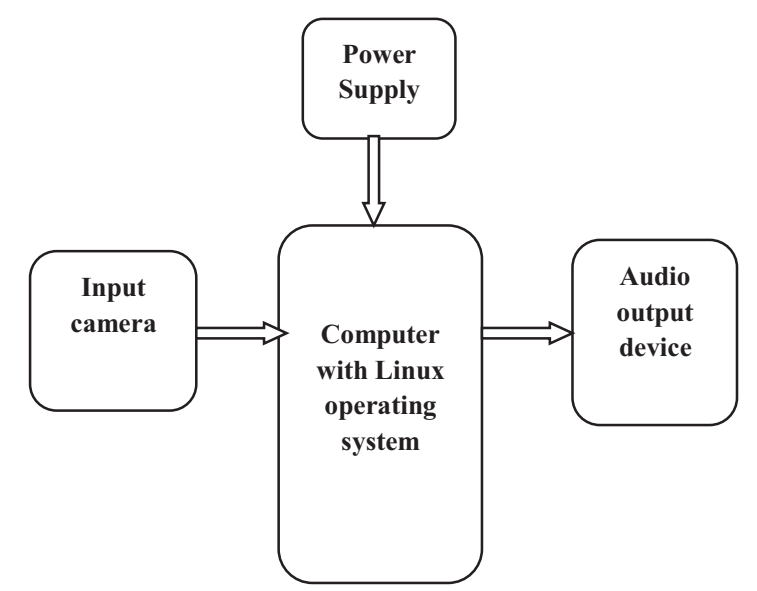

Figure 1. Block Diagram of color recognition system.

\section{USB Camera}

USB camera [4] is capable of capturing the highresolution image of cloth and which will be sent to recognizing the color [5].

\section{Audio Output Device}

LCD display can also be used to display the output but this scheme develops a device which is especially useful for visually impaired persons. So, In order to communicate with them, an audio output device plays an important role, which will act as a control surface, Human Interface Device (HID) which allows the user to control a digital audio or other digital audio application. Generally, this will contain one or more controls that can be assigned to the software, allowing control of the software. As digital audio software is complex and which can play a number of functions in the audio chain.

\section{METhodology OF COLOR RECOGNITION SYSTEM}

In this methodology, two types of classification are performed. The first type of classification is the computation of features and the second type of classification is to extract the features using suitable classifiers. In general, all the images used for this experiment are taken in natural light and then resized. 


\section{A. Model Conversion}

Color [6] is the most important feature of human eyes. By representation, this feature act as the overall of image content when it is used as a "global" feature. Space is defined as a model representing in terms depending on the values of intensity color space is defined as different models. Such as RGB, Lab, HSV, HSI, YCbCr, etc [7]. Though there are different color spaces each has its own benefits and drawbacks. In this paper, the input image has to be converted from one space to another. As all the images are in RGB model space, they should be changed into the suitable space. Among which HSV and $\mathrm{L}^{*} \mathrm{a}^{*} \mathrm{~b}$ are much likely to be used. The drawbacks that occur in RGB model is eliminated by using $\mathrm{L}^{*} \mathrm{a} * \mathrm{~b}$, HSV, HSI and YCbCr space models.

\section{B. RGB to $L * a * b$ model conversion}

According to the international standards, $1 * a * b$ is used for measurements, which has been used by the Commission International d'Eclairage (CIE) [8]. By using this model we can generate the image which consists of " $L$ " is the luminance or lightness component, which ranges from 0 to 100 , and as the parameters "a" (from green to red) and "b"(from blue to yellow) are the two chromatic components, which range from -120 to 120 .

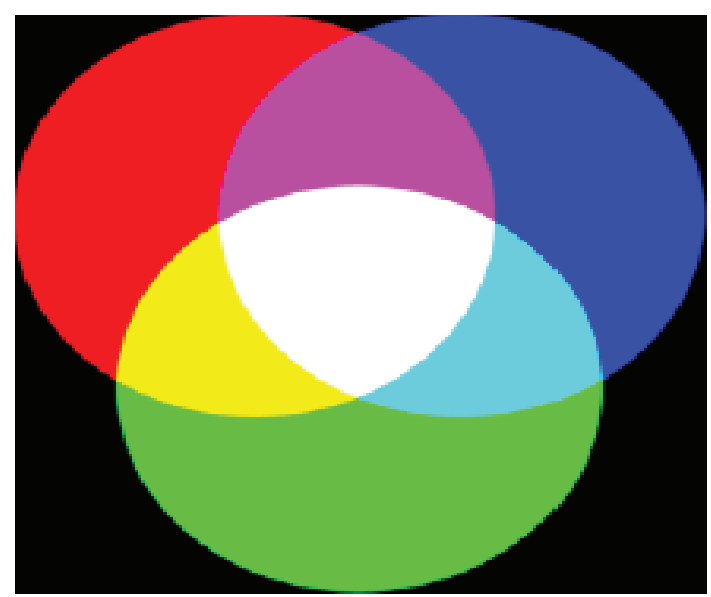

Figure 2. RGB additive primary model (RGB model)

\section{Identification of colors of clothes}

This is done based on the color histogram, the main theme of this is quantized the pixels of the image based on the relation between the $1 * a * b$ array of color space. If the camera-based [9] captured, input image has more colors then the dominant color is given as output.

\section{Color histogram}

This will give us information that how the colors are distributed in the image based on the pixel values. Pixels are arranged in RGB channels and this is represented by using the numerical values ranges from 0 255.Based on the count of pixel value each color is defined [10].
Let us consider an example of pure purple color image,

In the image every color has RGB channels; in the same way, this purple color has the value of RGB channel as shown in figure 3 .

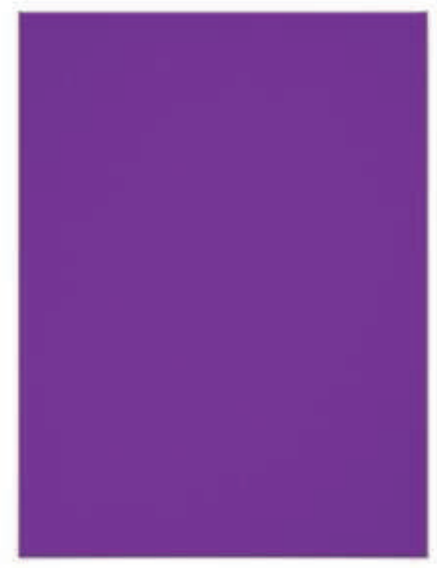

Figure 3. Purple [R G B] $=\left[\begin{array}{lll}118 & 52 & 149\end{array}\right]$

For the original image Figure 3 Purple (a), when the luminance scales of each RGB channels is increased by 30 . Then the color is transformed as shown in figure 4 .

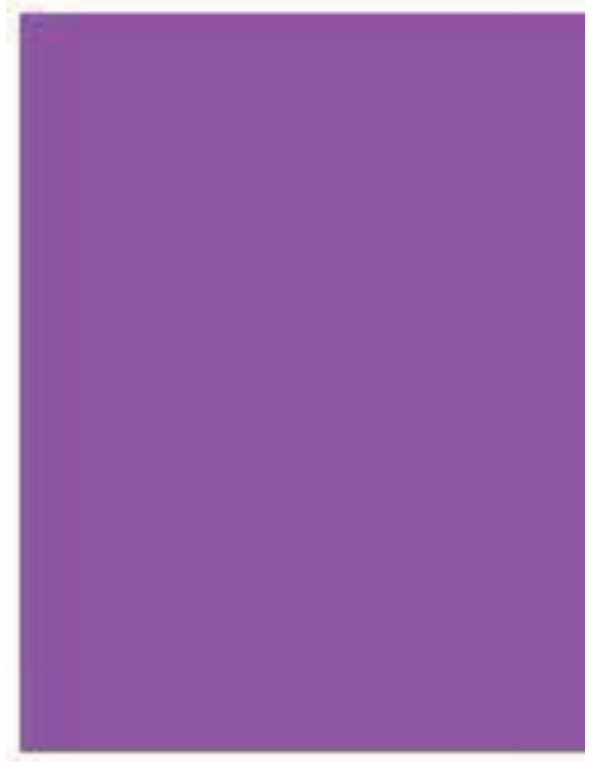

Figure 4. Purple [R G B] $=\left[\begin{array}{lll}148 & 82 & 179\end{array}\right]$

For the original image Figure 3 Purple (a), when the luminance scales of each RGB channels is increased by 40 . Then the color is transformed as shown in figure 5 . 


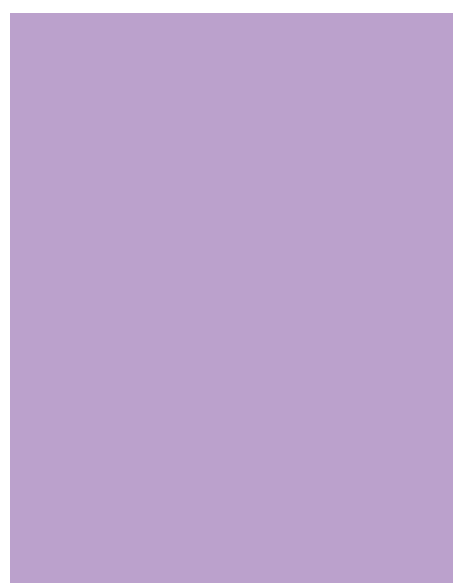

Figure 5. Purple [R G B] $=\left[\begin{array}{lll}188 & 62 & 219\end{array}\right]$

For the original image Figure 3 purple (a), when the luminance scales of each RGB channels is decreased by 10 . Then the color is transformed as shown in figure 6 .

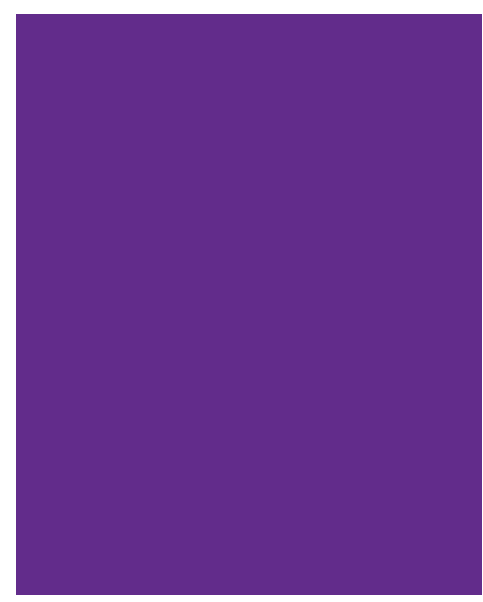

Figure 6. Purple [R G B] $=\left[\begin{array}{lll}98 & 42 & 139\end{array}\right]$

For the original image Figure 3 purple (a), When the luminance scale of each RGB channels is decreased by i.e., if only $G$ channel is made as 0 . Then the color is transformed as shown in figure 7.

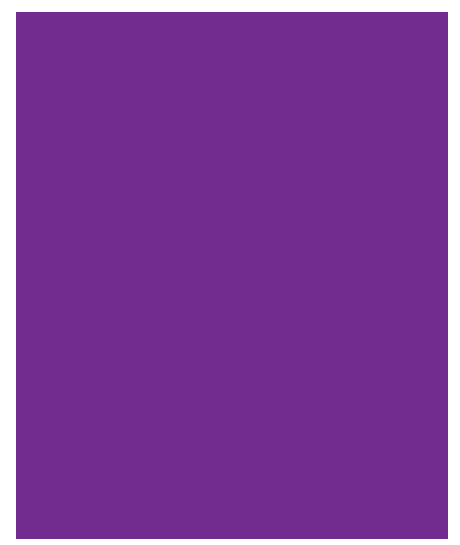

Figure 7. Purple [R G B] $=\left[\begin{array}{lll}118 & 0 & 149\end{array}\right]$

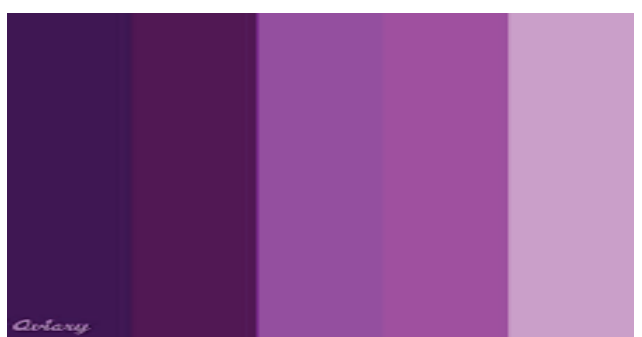

Figure 8. Different shades of purple

Above figure gives us the information about the different shades of purple color which can be obtained by changing RGB channel values.

\section{E. Color Labeler}

Color labeler program is used to tag image regions with a text label of a color. The first step in this paper is to create a python class that can be used to label shapes in an image with their associated color [3][11] to do this, defines a class named color labeler in the color labeler.py file:

$$
\begin{aligned}
& \text { "red": }(255,0,0), \\
& \text { "green": }(0,255,0), \\
& \text { "blue": }(0,0,255), \\
& \text { "pink": }(255,20,147), \\
& \text { "purple": }(90,40,200),
\end{aligned}
$$

To start this, color dictionary is used to the mapping of the color name (the key to the dictionary) to the RGB tuple (the value of the dictionary). From there, system allocate to memory to store these colors, followed by initializing the list of color names. The next step is to loop over the colors dictionary.

RGB channel of the input image is compared with color labeler and according to this; it gives the name of the color in the form of the text and then finally converted into speech [7].

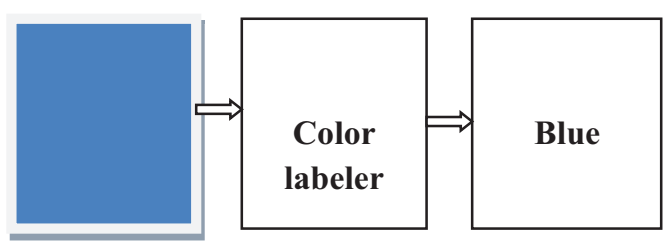

Figure 9. Output of color labeler

\section{Software Used}

Open CV (Open Source Computer Vision) is a library of programming functions mainly aimed at real-time computer vision. It has $\mathrm{C}++, \mathrm{C}$, Python and Java interfaces and supports Windows, Linux, Mac OS, ios, and Android.

Linux is one of the popular versions of UNIX Operating System. It is open source and easy to use. The Linux was designed considering UNIX compatibility. Its functionality list is quite similar to that of UNIX. 
Python is a widely used high-level programming language for general purpose programming. Python has a design philosophy that emphasizes code readability, and a syntax that allows programmers to express concepts in fewer lines of code that might be used in languages such as $\mathrm{C}++$ or Java. The language provides constructs intended to enable writing clear programs on both a small and large scale. Python interpreters are available for many operating systems, allowing Python code to run on a wide variety of systems. Python, the reference implementation of Python, is open source software and has a community-based development model, as nearly all of its variant implementations.

\section{WORKING}

This paper consist of three major steps which are as follows

1. Capture the image by using camera and saved it for further use.

2. Processing of saved image according to the algorithm and finding the color of the clothes. In this paper processing element is desktop computer or a portable computer like Raspberry.

3. Then the results are given to audio output device. By which the information i.e., about the colors of clothes is given to the visually impaired persons.

\section{A. Flowchart for Color Recognition}

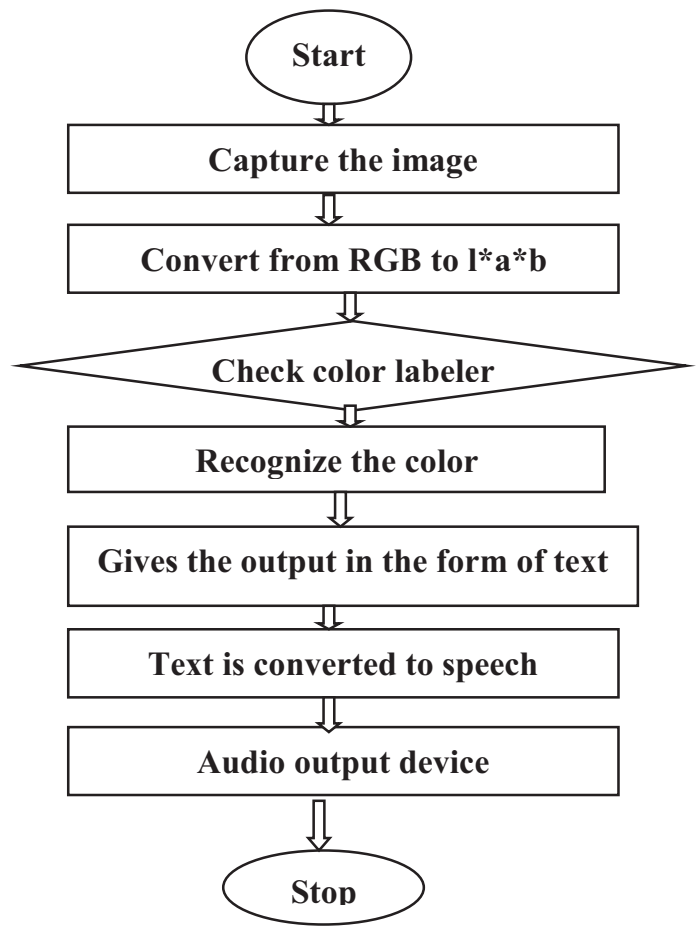

Figure 10. Flowchart for Color Recognition

\section{RESUlts}

The color [3] recognition device is used for identifying the colors of clothes and this will be useful for the visually impaired person. This device is developed by using open CV techniques along with some other methods. This will give the output in the form of audio. Here the below table will show the results through this device i.e., the color of clothes.

TABLE I.

RESULTS OF COLOR RECOGNITION SYSTEM

\begin{tabular}{|l|c|}
\hline CLOTHES & $\begin{array}{c}\text { OUTPUT AS SPEECH } \\
\text { (COLOR) }\end{array}$ \\
\hline & \\
\hline & \\
\hline & \\
\hline
\end{tabular}




\section{A. Advantages}

1. Low power consumption.

2. Fully automatic, fast response and user-friendly.

3. Low cost to design the circuit.

4. Efficient design.

5. Easy to operate.

This work can be extended by

1) Adding the feature of detecting and recognizing more types of indoor objects and icons on signage.

2) By using high-resolution cameras [12] and Bluetooth earphones can also be used for a clear and noise-free output to assist visually impaired persons to become independent.

\section{Conclusions}

This paper "Color recognition system for visually impaired persons" is proposed by using open CV on Linux Processor for identifying the colors of clothes. The paper is mainly intended for visually impaired persons as they are facing many problems in everyday life as everything is encoded visually. This research paper is a small contribution towards the task of bringing the visually impaired people into the environment by providing an independent device to them.

\section{REFERENCES}

[1] Mr.Shashi kumar, Mr. Mohamed zakir, Mr. Nikhil kumar. Sai Vidya Institute of Technology, Bangalore "Assistive clothing pattern recognition for visually impaired people" Project reference No:38S0161.

[2] Sameer Antania, Rangachar Kasturia; Ramesh Jain "A survey on the use of pattern recognition methods for Abstraction, indexing and retrieval of images and video", Pattern Recognition 35 (2002) 945-965.
[3] Xiaodong Yang, Shuai Yuan, andyinglitian, "Assistive Clothing Pattern Recognition for Visually Impaired People," IEEE transactions on human machine systems, vol. 44, NO. 2, APRIL 2014.

[4] S. Liu, J. Feng, Z. Song, T. Zhang, H. Lu, C. Xu, and S. Yuan, in Proc. "Hi, magic closet, tell me what to wear," ACM Multimedia, 2012.

[5] Irati Rasines1, Pedro Iriondo2, and Ibai Díez, K. Miesenberger et al. (Eds.): "Real - Time Display Recognition System for Visually Impaired" ICCHP 2012, Part II, LNCS 7383, pp. 623-629, 2012. (C) Springer-verlagberlin Heidelberg 2012.

[6] N Durga Rao, Dr. G Sudhavani, P Balakrishna, Dr. K Gouthami, "Cloth Pattern Recognition With Four Features (RSSM)", 978-1-4673-6708-0/15/C2015 IEEE.

[7] Ibrahim Patel, Jagdish Goud "Color Recognition for Blind and Color Blind People". International Journal of Engineering and Innovative Technology (IJEIT) Volume 2, Issue 6, December 2012

[8] Shruti Bharadwaj, Sharath H.K., Praveena M.B.,Ajay Shetty,Shivarudraiah B."Clothing Color and Pattern Recognition for Visually Impaired People". International Journal of Engineering, Management \& Sciences (IJEMS) ISSN-2348-3733, Volume-2, Issue-5, May 2015.

[9] Honey Mishra "The Survey Paper on Clothing Pattern and color recognition for Visually Impaired People", ,IJSRD International Journal for Scientific Research \& Development| Vol. 3, Issue 09, 2015 | ISSN(online): 2321-0613.

[10] Thogaricheti Ashwini, Mrs. Anuradha.S.G. "Assistive clothing Pattern Recognition for Impaired people". International Journal of Advanced Research in Computer and Communication Engineering Vol. 5, Issue 4, April 2016

[11] J. Zhang, M. Marszalek, S.Lazebnik, and C. Schmid, "Local features and kernels for classification of texture and object categories: A comprehensive study," Int. J. Computer. Vis., vol. 73, no. 2, pp. 213-238, 2007.

[12] Rakhil k s , Asha s, "Improved fabric defect detection and pattern Classification using radon transform" International journal of innovative research in Science, engineering and technology, vol. 4, issue 7, July 2015. 